\title{
Meeting report: The biology of genomes and proteomes
}

\author{
Kyudong Han ${ }^{1} \cdot$ Han Jun $\mathrm{Jin}^{2} \cdot$ Heui-Soo $\mathrm{Kim}^{3} \cdot \mathrm{Nam}-\mathrm{Soo} \mathrm{Kim}^{4}$
}

Received: 1 June 2015/ Accepted: 2 June 2015/Published online: 6 June 2015

(C) The Genetics Society of Korea and Springer-Science and Media 2015

A mini-symposium of the Genetics Society of Korea was held at Dankook University, Korea on April 24, 2015. The theme of the mini-symposium was 'The biology of genomes and proteomes.' This was the first mini-symposium of 2015. There were two sessions that were chaired by Dr. Kyu-Dong Han (Dankook University, Korea) and Dr. Heui-Soo Kim (Pusan National University, Korea).

In the first session, three speakers gave talks on genomics and epigenomics. The first speaker, Dr. Keunsoo Kang (Department of Microbiology, Dankook University, Korea), introduced "An automated analysis pipeline for a large set of ChIP-seq data: AutoChIP (Kim et al. 2014a, b)." Recent advances in genomic DNA sequencing have revolutionized the ways to examine molecular events inside cells in several aspects. Next-generation sequencing approaches, which enable the rapid and accurate sequencing of short DNA fragments, have changed the ways researchers approach biological problems. For example, researchers can identify the genome-wide binding sites of proteins by using chromatin immune precipitation coupled with parallel sequencing (ChIP-seq) without any prior

Nam-Soo Kim

kimnamsu@kangwon.ac.kr

1 Department of Nanobiomedical Science and BK21 PLUS NBM Global Research Center for Regenerative Medicine, Dankook University, Cheonan 330-714, Republic of Korea

2 Department of Nanobiomedical Science, Dankook University, Cheonan 330-714, Republic of Korea

3 Department of Biological Sciences, College of Natural Sciences, Pusan National University, Busan 609-735, Republic of Korea

4 Department of Molecular Bioscience, Institute of Bioscience and Biotechnology, Kangwon National University, Chuncheon 200-701, Korea knowledge (Johnson et al. 2007; Robertson et al. 2007). Although there are many applications available for the analysis of ChIP-seq data (Henry et al. 2014), users need to know some details about the installation, alignment, and peak-calling procedures in most cases. Dr. Kang summarized his recent advances with the AutoChip assay, which is an easy-to-use application for ChIP-seq analysis. Using the AutoChip, the user automatically downloads and installs programs on his or her computer before launching the actual data analysis. In addition, all procedures for ChIP-seq analyses, such as the alignment of unmapped reads to a reference genome and the identification of genome-wide binding sites for a given protein, can be done by two simple steps coupled with AutoChIP. An evaluation of the cocktail algorithm implemented in AutoChIP showed that the algorithm outperformed a single ChIP-seq tool in terms of the ratio of motif occurrences and the average height of the normalized read density over the identified peaks. In addition, the annotation of the identified peaks with information about known genes and repeat elements provides a comprehensive picture of the genome-wide binding sites of selected proteins. Overall, AutoChIP provides a comprehensive platform to analyze a large set of ChIPseq data in one step.

The second speaker, Dr. Kyunghwan Kim (Department of Biology, Chungbuk National University, Korea), gave a presentation entitled 'Epigenetics and cancer development.' The regulation of gene expression and the maintenance of chromosome stability in eukaryotes are critically associated with the formation of silent chromatin. Inactive chromatin structures are frequently related to distinctive histone modifications (Suganuma and Workman 2011). Although recent studies have implicated histone modification as a key regulator of chromatin fluidity, the molecular mechanisms underlying such effects remain 
largely unknown. Dr. Kim's previous studies revealed that VprBP acts as an effector that binds histone $\mathrm{H} 3$ tails protruding from nucleosomes and establishes chromatin silencing in cancer cells (Kim et al. 2012a, b). Those results clearly pointed out that VprBP has a negative regulatory role in transcription. Nonetheless, precisely how VprBP mediates its effects on the formation of repressive chromatin domains is poorly understood. In his talk, Dr. Kim extensively provided solid evidence that VprBP has an intrinsic kinase activity and can phosphorylate histone $\mathrm{H} 2 \mathrm{~A}$ at T120. H2AT120 phosphorylation (H2AT120p) by $\mathrm{VprBP}$ is functionally critical, because a point mutation at H2AT120 eliminates the observed effects of VprBP. Mechanistic studies revealed that $\mathrm{VprBP}$ is recruited to a large set of tumor suppressor genes and establishes an H2AT120p mark, resulting in gene silencing. In accordance with those results, tumor array experiments demonstrate a clear link between the elevated expression of VprBP and increased levels of H2AT120p. Of equal importance, Dr. Kim identified a highly potent and selective inhibitor of $\mathrm{VprBP}$ that interferes with cell proliferation and tumorigenesis. He has conducted a rather comprehensive investigation of VprBP-mediated H2AT120p, supporting the potential significance of that epigenetic pathway in human cancer. A large number of devastating human cancers result from a failure to properly control gene expression due to aberrant chromatin regulation. Dr. Kim's data demonstrate VprBP as a new histone kinase and uncover the mechanisms underlying VprBPmediated gene repression in cancer cells.

The third speaker, Dr. Kwonho Hong (Department of Nanobiomedical Science, Dankook University, Korea), discussed a critical role for mouse Dot1L in vessel development. Studies suggest that the precise regulation of genome-wide chromatin structure ensures the proper spatial and temporal expression patterns of many genes involved in diverse biological processes. One of the major questions emerging in modern vascular biology is how chromatin dynamics modulate vessel-wall homeostasis in both physiological and pathological conditions. Although studies have suggested that the aberrant regulation of histone modifications is involved in certain types of cardiovascular anomalies, in vivo evidence of the cellular mechanism(s) of the disorders remains unclear, particularly with respect to the role of histone methylation. Dr. Hong's previous genetic studies demonstrated that mouse Dot1L also plays a pivotal role in early mouse development. Homozygous mutation of Dot $1 \mathrm{~L}$ causes embryonic lethality by E10.5 with cardiovascular defects including the enlargement of heart chambers, poorly developed vasculatures, dilated vessel lumina, and hemorrhages (Jones et al. 2008; Nguyen et al. 2011). Until now, it has been unclear whether the vascular phenotypes observed in
Dot $1 L-/-$ mice are a cell-autonomous function of Dot1L in endothelial cells (ECs). Therefore, Dr. Hong attempted to disrupt Dot1L in ECs by crossing a Dot1L conditional allele with a $\mathrm{Tg}$ (Tie2-Cre) strain. The conditional Dot1L knockout exhibited lethality during late gestation (E14.5 P1). All mutants showed severe hemorrhage on the skin and brain. Immunohistochemistry analysis revealed dilated blood vessels in the skin and brain. Aberrant tube formation was also observed in Dot1Lknockdown HUVECs. Finally, Dot1L deficiency prohibited the differentiation of CD31-positive ECs in Dot1Lknockdown mouse embryonic stem cells. In conclusion, Dr. Hong's data suggest that ECs are the cell type responsible for the vascular phenotype displayed in conventional Dot1L-knockout mice.

In the second session, three speakers gave talks on network biology, fish genomics, and metabolomics. The first speaker, Dr. Sanguk Kim (Department of Life Science, Pohang University of Science and Technology, Korea), gave a presentation entitled 'Connecting genotype to phenotype through network biology and protein functional modules.' Recently, several genetic variants associated with human diseases have been identified through advances in large-scale sequencing techniques (Meyerson et al. 2010; Boycott et al. 2013). The detection of disease-causing gene variants in human subjects suffers, however, from the incomplete penetration of environmental variation, as well as the diverse genetic background of the human population (Cooper et al. 2013). Thus, the accurate genotype-phenotype maps of model organisms are important for understanding the relationship between diseases and associated genes. Researchers have been debating whether heritable phenotypes measured in model organisms can facilitate the identification of genes associated with human diseases. It has been assumed that genes labeled as essential could be associated with human diseases, because perturbations of such genes exhibit discernible phenotypes. However, mutations in many nonessential genes have also shown associations with multiple human diseases (Goh et al. 2007). Dr. Kim's group attempted to establish a relationship between human diseases and heritable phenotypes measured directly from a model organism. His presentation highlighted the finding that a genotype-phenotype map produced in a model organism can estimate the progression of human disease based on genetic variation. Furthermore, alterations of gene essentiality are fundamental for organismal evolution. It is not yet clear, however, how the essentiality of orthologs diverges across species. Using network evolution and comparative genomic analysis, Dr. Kim's research team studied changes in gene essentiality between yeast and mice ( Kim et al. 2012a). His study describes how network evolution changes gene essentiality. 
The second speaker, Dr. Woo-Jin Kim (Biotechnology Research Division, National Fisheries Research and Development Institute, Korea), talked about the 'Wholegenome sequence of olive flounder and its application.' The olive flounder (Paralichthys olivaceus) is one of the major cultured fishes and is one of the most important commercial fishes. Hence, it is necessary to establish the whole-genome sequence of $P$. olivaceus and to develop molecular markers related to economically important features, such as growth and disease resistance, to maintain a dominant position in the flounder aquaculture industry. In general, females have high economic value due to their faster growth compared with that of males. Therefore, if technology for female-only seeding production is developed, we can expect cost reduction and improved productivity for fish farms. Dr. Kim presented his study to identify sex-related molecular markers through the whole-genome sequencing of the olive flounder. The whole genome $(\sim 550 \mathrm{Mb})$ of the olive flounder was sequenced to $40 \times$ coverage using FLX-454. More than $535 \mathrm{Mb}$ of sequence ( $>97 \%$ coverage) was generated with 1581 scaffolds $(\mathrm{N} 50=1.12 \mathrm{Mb})$ and 256,098 contigs $(N 50=2985$ bp). Dr. Kim's research team constructed a high-resolution genetic map composed of 24 linkage groups by using 1518 microsatellites. In addition, the 1581 scaffolds were arranged into 24 chromosomes, and their transposable element composition was analyzed. Transposable elements occupy approximately $5.85 \%$ $(29.6 \mathrm{Mb})$ of the entire genome, and retroelements, DNA transposons, and satellites occupy 1.66, 0.97, and $0.06 \%$, of the entire genome, respectively. The libraries were constructed in the EcoRI and HindIII sites of the vector CopyControl pCC1BAC. The two libraries contained a total of 66,816 BAC clones arrayed in one-hundred seventy-four 384-well microtiter plates that correspond to 14.18 haploid genome equivalents based on the olive flounder genome size of $550 \mathrm{Mb}$. A random sampling of $335 \mathrm{BACs}$ indicated an average insert length of $118 \mathrm{~kb}$ with a range of $80-180 \mathrm{~kb}$, while $1.5 \%$ of the BACs did not contain inserts. The combined libraries have a greater than $99 \%$ probability of containing any single-copy sequence. The BAC library has been arranged in three-dimensional pools, allowing screening with various PCR-based markers. A total $13.65 \mathrm{~Gb}$ of transcriptome sequence was established for gene annotation, and 40,691 protein coding genes were annotated using ab initio gene prediction, protein homology gene prediction, and consensus gene-structure annotation. To effectively provide that information to users, all of the information including the whole-genome sequence with scaffolds, BES, ESTs, repeat elements, and comparisons with genes and ESTs of closely related species was stored in a genome web browser. Based on those data, Dr. Kim's group identified and validated a sex-determining marker. Through a genome-wide association study of males and females, they identified 11 SNP markers located on chromosome 9 that showed a significant difference between males and females $(\mathrm{P}<0.001)$. In an analysis of the sexassociation SNPs among four groups (gynogenetic diploids, males, pseudomales, and females), a homozygous SNP was detected in all of the females, gynogenetic diploids, and pseudomales, which are genetically the same as females. A heterozygous SNP was not detected in all of the males, however, implying that some males had changed their sex from female due to high water temperature during sexual differentiation (Yamamoto 1999). In addition, some genes that overlapped with the SNP were expressed increasingly in the testes before sexual differentiation. Thus, Dr. Kim could guess that those genes are closely related to sex determination. Based on that, Dr. Kim's group expects to develop a molecular biomarker to distinguish males and pseudomales, resulting in all-female production by breeding pseudomales and females. In the report, they identified a sex-associated biomarker for the olive flounder as a good example of the application of whole-genome sequencing.

The third speaker, Dr. Suhkmann Kim (Department of Chemistry and Center for Proteome Biophysics, Pusan National University, Korea), reported on the 'Metabolomics study of phenotype discrimination for several different applications.' Metabolomics is a part of systems biology and has great potential for finding biomarker groups for disease diagnosis and toxicity assessment (Gowda et al. 2008). Recent developments in NMR allow researchers to measure gel or semi-solid samples with HRMAS (High Resolution-Magic Angle Spinning) or nanoprobes (Li et al. 2011). In Dr. Kim's study, tissues and cells were used for $1 \mathrm{D}$ proton measurement without pre-treatment using nano-probes. The method showed a resolution similar to that of a solution 1D spectrum and had a much faster total analysis time. The technique could also be applied to serum or blood samples, because those samples have a high viscosity in some cases. Dr. Kim discussed several applications of NMR-based metabolomics studies, including disease diagnosis, measuring the toxicity of chemicals, forensic applications, and environmental toxicology. The method includes different kinds of samples including human and animal urine, serum, and tissue and cultured cells. In a first application, Dr. Kim tried to distinguish colorectal cancer and normal tissue using HRMAS NMR techniques (Kim et al. 2013b). The cancer tissue showed higher levels of arginine, betaine, glutamate, lysine, and taurine and lower levels of glutamine, hypoxanthine, isoleucine, lactate, methionine, pyruvate, and tyrosine relative to normal tissue. In another example, Dr. Kim carried out HR-MAS NMR experiments to distinguish metabolic changes in human hepatocellular carcinomas (HCCs) and colorectal liver metastases (CRLMs) and then confirmed that each tissue sample was clearly classified 
into different groups by multivariate analysis (Kim et al. 2014a). Good separation among tumors and non-tumorous hepatic parenchyma was observed in OPLS-DA loading plots. In addition, the metabolomic characteristics of HCCs showed marked separation from those of CRLMs by OPLS-DA. Compared with CRLM, HCC showed elevated levels of glucose, glutamine, o-phosphocholine, and snGlycero-3-phosphocholine $(\mathrm{P}<0.05)$ and decreased levels of hypoxanthine $(\mathrm{P}=0.01)$. Dr. Kim will confirm the metabolic pathways causing those differences in future work. For the forensic applications, he analyzed urine from an individual with a history of sniffing glue using NMRbased metabolomics (Kwon et al. 2011). Toluene is the main solvent ingredient of glue, but it is not soluble in water and is difficult to detect in biofluid. From his study, Dr. Kim found an enormous hippuric acid peak in the test individual's urine. Inhaled toluene is metabolized to hippuric acid in the liver and excreted in the urine. In another example, Dr. Kim found that the biomarker ethyl glucuronide could be used instead of ethanol as an indicator of alcohol consumption (Kim et al. 2013a). Dr. Kim applied NMR-based metabolomics to tests of environmental toxicity and successfully found many biomarkers in zebrafish for each chemical treatment tested, including organic solvents, pesticides, and medicines (Yoon et al. 2013). Dr. Kim's presentation demonstrated the usefulness and versatility of NMR spectroscopy coupled with multivariate analysis in investigating the metabolic responses to external stimuli and in identifying the responsible individual metabolites and/or metabolic pathways.

\section{References}

Boycott KM, Vanstone MR, Bulman DE, MacKenzie AE (2013) Rare-disease genetics in the era of next-generation sequencing: discovery to translation. Nat Rev Genet 14:681-691

Cooper DN, Krawczak M, Polychronakos C, Tyler-Smith C, KehrerSawatzki H (2013) Where genotype is not predictive of phenotype: towards an understanding of the molecular basis of reduced penetrance in human inherited disease. Hum Genet 132:1077-1130

Goh KI, Cusick ME, Valle D, Childs B, Vidal M, Barabasi AL (2007) The human disease network. Proc Natl Acad Sci USA 104:8685-8690

Gowda GA, Zhang S, Gu H, Asiago V, Shanaiah N, Raftery D (2008) Metabolomics-based methods for early disease diagnostics. Expert Rev Mol Diagn 8:617-633
Henry VJ, Bandrowski AE, Pepin AS, Gonzalez BJ, Desfeux A (2014) OMICtools: an informative directory for multi-omic data analysis. Database (Oxford). doi:10.1093/database/bau069

Johnson DS, Mortazavi A, Myers RM, Wold B (2007) Genome-wide mapping of in vivo protein-DNA interactions. Science 316:1497-1502

Jones B, Su H, Bhat A, Lei H, Bajko J, Hevi S, Baltus GA, Kadam S, Zhai H, Valdez R et al (2008) The histone H3K79 methyltransferase Dot1L is essential for mammalian development and heterochromatin structure. PLoS Genet 4:e1000190

Kim K, Heo K, Choi J, Jackson S, Kim H, Xiong Y, An W (2012a) Vpr-binding protein antagonizes p53-mediated transcription via direct interaction with $\mathrm{H} 3$ tail. Mol Cell Biol 32:783-796

Kim J, Kim I, Han SK, Bowie JU, Kim S (2012b) Network rewiring is an important mechanism of gene essentiality change. Sci Rep 2:900

Kim S, Lee M, Yoon D, Lee D-K, Choi H-J, Kim S (2013a) 1D proton NMR spectroscopic determination of ethanol and ethyl glucuronide in human urine. Bull Korean Chem Soc 34:2413-2418

Kim S, Lee S, Maeng YH, Chang WY, Hyun JW, Kim S (2013b) Study of metabolic profiling changes in colorectal cancer tissues using 1D1H HR-MAS NMR spectroscopy. Bull Korean Chem Soc 34:1467-1472

Kim T, Lee W, Han K, Kang K (2014a) An automated analysis pipeline for a large set of ChIP-seq data: AutoChIP. Genes Genom 37:305-311

Kim JM, Kwon CH, Joh JW, Park JB, Lee JH, Kim SJ, Paik SW, Park CK, Yoo BC (2014b) Differences between hepatocellular carcinoma and hepatitis B virus infection in patients with and without cirrhosis. Ann Surg Oncol 21:458-465

Kwon B, Kim S, Kim S, Lee DK, Park YJ, Kim MD, Lee JS, Kim S (2011) 1H NMR spectroscopic identification of a glue sniffing biomarker. For Sci Int 209:120-125

Li M, Song Y, Cho N, Chang JM, Koo HR, Yi A, Kim H, Park S, Moon WK (2011) An HR-MAS MR metabolomics study on breast tissues obtained with core needle biopsy. PLoS ONE 6:e25563

Meyerson M, Gabriel S, Getz G (2010) Advances in understanding cancer genomes through second-generation sequencing. Nat Rev Genet 11:685-696

Nguyen AT, Xiao B, Neppl RL, Kallin EM, Li J, Chen T, Wang DZ, Xiao X, Zhang Y (2011) DOT1L regulates dystrophin expression and is critical for cardiac function. Genes Dev 25:263-274

Robertson G, Hirst M, Bainbridge M, Bilenky M, Zhao Y, Zeng T, Euskirchen G, Bernier B, Varhol R, Delaney A et al (2007) Genome-wide profiles of STAT1 DNA association using chromatin immunoprecipitation and massively parallel sequencing. Nat Methods 4:651-657

Suganuma T, Workman JL (2011) Signals and combinatorial functions of histone modifications. Ann Rev Biochem 80:473-499

Yamamoto E (1999) Studies on sex-manipulation and production of cloned populations in hirame, Paralichthys olif́aceus (Temminck et Schlegel). Aquaculture 173:235-246

Yoon D, Lee M, Kim S, Kim S (2013) Applications of NMR spectroscopy based metabolomics: a review. J Korean Magn Reson Soc 17:1-10 